W Von Hans Holzinge as wir uns als zukunftsmöglich vorstellen können, hängt wesentlich davon $a b$, wie wir die Gegenwart wahrnehmen. Meist bestimmen eingefahrene Sichtweisen unser Denken. Neue Lösungen erfordern daher auch neue Sichtweisen der Probleme. Denn: Wer die Deutungsmacht über die Wirklichkeit hat, hat auch die Macht zur Veränderung.

Als Österreicher maße ich mir nicht an, die gegenwärtige Politik der rot-grünen Koalition in Deutschland zu bewerten. Auffällig erscheinen mir jedoch Parallelen zu Österreich in den Mustern, wie die Debatten über Strukturreformen, Arbeitslosigkeit und soziale Sicherung ablaufen und wie ökologische Themen von der Bildfläche verschwinden. Der ritualisierte Schlagabtausch zwischen Regierung und Opposition gleicht sich aufs Haar - die Opposition bezichtigt die Regierung, Belastungspakete über die Bevölkerung zu bringen, an der Steuerschraube zu drehen, Schuld an der steigenden Arbeitslosigkeit und am abfallenden Wirtschaftswachstum zu sein. Nachhaltigkeit spielt dabei keine Rolle mehr - sie verblasst zum Schönwort für Sonntagsreden.

Warum sind tatsächliche Strukturreformen so schwer durchsetzbar? Warum ist die Frage von Verteilungsgerechtigkeit derart tabuisiert? Und warum sind Umweltthemen völlig an den Rand gedrängt? Die Antworten lassen sich nicht anhand der verbreiteten politischen Parolen geben, sondern liegen tiefer. Die Denkzettelwahlen in Hannover und Hessen sind somit auch nicht die Lösung, sondern vielmehr Symptom beziehungsweise Teil der Probleme eines reduktionistischen politischen Diskurses.

Dem konservativen österreichischen Bundeskanzler wurde im Wahlkampf von der Opposition ,,soziale Kälte“ vorgeworfen, von „Hungerkuren“ oder gar „Blut, Schweiß und Tränen“, die seine Politik des Sparens über die Bevölkerung gebracht hätten, war die Rede. Und dennoch wurde er mit großer Mehrheit wieder gewählt. Der Angstwahlkampf ging ins Leere, weil er nicht den empirischen Fakten entsprach. Die österreichische Gesellschaft lebt in historisch einmaligem Reichtum. Das gilt auch für die bundesdeutsche. Zynisch war daher wohl die Kampagne vom ,letzten Hemd“. Das „letzte Hemd“ ziehen andere aus in der Welt und nicht die BürgerInnen eines der reichsten Länder der Welt. Die Art, wie die politischen Debatten derzeit geführt werden, entspricht dem, was Ulrich Beck treffend als Wohlstandsnationalismus bezeichnet. Kaum vorstellbar,
Politik im Wohlstandsnationalismus

\section{Diskursfalle überwinden}

\section{Der politisch vorherrschende Diskurs gegenseitiger Schuldzuweisung ver- hindert Reformen im Sinne sozialer und ökologischer Nachhaltigkeit und ver- weist auf das Mehrheitsdilemma der Demokratie in Wohlstandsgesell- schaften. Jene, die gut im Boot sitzen, verteidigen ihre Pfründe. Die sozial Benachteiligten, aber auch die Umwelt geraten dabei unter die Räder.}

dass etwa Fragen der Entwicklungszusammenarbeit eine Chance hätten, zum Thema von Wahlkämpfen zu werden.

Ich leugne nicht, dass es neue Armut und soziale Ausgrenzungen auch in den europäischen Reichtumsgesellschaften gibt. Doch diese werden vom politischen Diskurs immer weniger erfasst. So waren die Hartz-Vorschläge und die ihnen folgenden Debatten zum Thema Arbeitslosigkeit hauptsächlich auf Fragen der besseren Vermittlung Arbeitsloser konzentriert, als wären diese selber Schuld an der Arbeitslosigkeit. Der Appell an die Solidarität greift in Wohlstandsgesellschaften immer weniger, worauf John Kenneth Galbraith bereits vor Jahren hingewiesen hat: Die „Mehrheit der Wohlhabendengemeinde" sei immer weniger bereit, ihren sozialen (Steuer)-Beitrag zur Förderung von Benachteiligten zu leisten. Auf die Wohlstandsdemokratien kommt damit eine doppelte Herausforderung zu: Jene am unteren Ende der Gesellschaft können nicht nur ihre Interessen am wenigsten artikulieren; sie sind bei Wahlen immer auch in der Minderheit.

Auch Umweltpolitik scheint in der Wohlstandsgesellschaft immer schwieriger zu werden. Jeder will zwar eine intakte Umwelt, aber niemand die hierfür notwendigen Grenzen akzeptieren. Die von den österreichischen Grünen im Wahlkampf ohnedies sehr verhalten eingebrachte stärkere Ressourcenbesteuerung wurde von allen anderen Fraktionen diffamiert. Mit Rücksicht auf die „Mehrheitspartei der AutofahrerInnen“ wurde so getan, als hätten die zunehmenden Wetterka- priolen nichts mit dem menschengemachten Klimawandel zu tun, ganz abgesehen von den internationalen Verpflichtungen zur Kohlendioxidminderung, die Österreich und Deutschland eingegangen sind. Die Mini-Ökosteuer in Deutschland ist ja ebenso heftig unter Beschuss.

\section{Gibt es Auswege?}

„Gesamtwirtschaftlich sind Waren, Kapital und Erwerbsarbeitskräfte nicht mehr knapp. Sie sind in Fülle vorhanden. Doch das Denken ist noch immer in der Knappheitsuiberwindung verhaftet", schreibt die Ökonomin Luise Gubitzer. Der allseits das Denken und Fühlen bestimmende Knappheitsdiskurs verstellt den Blick auf alternative Problemsichten und Lösungen.

Es soll nicht geleugnet werden, dass Reformen im Gesundheits- oder Rentensystem anstehen. Auch kann die steigende Arbeitslosigkeit nicht bagatellisiert werden. Doch nur wenn es in $\mathrm{Zu}$ kunft gelingt, neue Wohlstandsbilder in die Diskussion zu bringen, wird wieder Bewegung in die Politik kommen. Nur wenn Fragen einer umfassenden Lebensqualität wie etwa der Abbau von Zeit- und Arbeitsstress, mehr Überschaubarkeit und Möglichkeiten der Partizipation in den Mittelpunkt treten, eröffnen sich auch Spielräume für Sozial- und Umweltpolitik. Nur dann kann ernsthaft vom Bemühen um nachhaltige Entwicklung gesprochen werden.

Nur wenn wir den Menschen Sinnangebote jenseits des verengenden Konsumismus bieten, sind sie bereit, eine solidarische Umverteilung von Arbeit und Erwirtschaftetem mit zu tragen. Offene Debatten über den Wandel von der ,Güteranhäufungsgesellschaft“ zur „Bildungs-, Kultur- und Beziehungsgesellschaft" wären demnach der Schlüssel für eine neue Politik der Solidarität in Reichtumsgesellschaften. Nachhaltigkeit, die Gerechtigkeit zwischen den Generationen fordert, wird somit Fragen der Verteilungsgerechtigkeit innerhalb der heute lebenden Generation - in der nationalen Gesellschaft wie in der Weltgesellschaft - ins Zentrum rücken müssen.

\section{Der Autor}

Hans Holzinger ist wiss. Mitarbeiter der RobertJungk-Bibliothek für Zukunftsfragen in Salzburg und Mitherausgeber der Zeitschrift "pro ZUKUNFT". Kontakt: Tel. 0043-662-873206,

E-Mail: jungk-bibliothek@salzburg.at 
(c) 20I0 Authors; licensee IÖW and oekom verlag. This is an article distributed under the terms of the Creative Commons Attribution Non-Commercial No Derivates License (http://creativecommons.org/licenses/by-nc-nd/3.o/), which permits unrestricted use, distribution, and reproduction in any medium, provided the original work is properly cited. 